\title{
On Choosing Effective Elasticity Tensors Using a Monte-Carlo Method
}

\author{
Tomasz DANEK $^{1,2}$ and Michael A. SLAWINSKI ${ }^{1}$ \\ ${ }^{1}$ Department of Earth Sciences, Memorial University of Newfoundland, \\ St. John's, Canada; e-mail: mslawins@mun.ca \\ ${ }^{2}$ Department of Geoinformatics and Applied Computer Science, \\ AGH - University of Science and Technology, Kraków, Poland \\ e-mail: tdanek@agh.edu.pl (corresponding author)
}

\begin{abstract}
A generally anisotropic elasticity tensor can be related to its closest counterparts in various symmetry classes. We refer to these counterparts as effective tensors in these classes. In finding effective tensors, we do not assume a priori orientations of their symmetry planes and axes. Knowledge of orientations of Hookean solids allows us to infer properties of materials represented by these solids. Obtaining orientations and parameter values of effective tensors is a highly nonlinear process involving finding absolute minima for orthogonal projections under all three-dimensional rotations. Given the standard deviations of the components of a generally anisotropic tensor, we examine the influence of measurement errors on the properties of effective tensors. We use a global optimization method to generate thousands of realizations of a generally anisotropic tensor, subject to errors. Using this optimization, we perform a Monte Carlo analysis of distances between that tensor and its counterparts in different symmetry classes, as well as of their orientations and elasticity parameters.
\end{abstract}

Key words: anisotropy, vertical seismic profile (VSP), inversion.

Ownership: Institute of Geophysics, Polish Academy of Sciences;

(C) 2015 Danek and Slawinski. This is an open access article distributed under the Creative Commons Attribution-NonCommercial-NoDerivs license,

http://creativecommons.org/licenses/by-nc-nd/3.0/. 


\section{INTRODUCTION}

The purpose of this paper is to examine a generally anisotropic elasticity tensor, expressed in terms of twenty-one elasticity parameters and obtained from vertical seismic profiling (VSP) measurements, to infer properties of materials represented by this tensor. Beginning with a generally anisotropic tensor, we are able to infer these properties by relating this tensor to its closest counterparts in the sense of Frobenius norm, as defined by Gazis et al. (1963), in all material symmetries of Hookean solids, as shown by Danek et al. (2013). Herein, we focus our attention on examining symmetries used in seismology: monoclinic, orthotropic, transversely isotropic, and isotropic tensors. Following the definition and nomenclature of Kochetov and Slawinski (2009a, b), we refer to these counterparts as effective tensors of these symmetry classes. Note that we use generally anisotropic and orthotropic, not triclinic, and orthorhombic crystals, respectively. The latter terms are associated with lattice symmetries of crystals, while in seismology we deal with continua and their symmetries, which are symmetries of the elasticity tensor. In such a case, orthotropic refers to three mutually orthogonal symmetry planes.

Consideration of a generally anisotropic tensor allows us to examine effective tensors belonging to distinct symmetry classes without a bias of prior assumptions. In other words, the choice of the symmetry class model is guided by the data from which the generally anisotropic tensor is derived.

Obtaining these orientations and parameter values is a mathematically involved process. Explicit underpinnings of the methodology used in this paper are presented by Danek et al. (2013), and the reader is referred to that publication and references therein. Herein, we provide an overview to render the present paper self-contained.

To infer information about materials examined through VSP measurements, we consider relationships between the obtained tensor and its symmetric counterparts. Such a tensor was obtained by Dewangan and Grechka (2003) from multi-component and multi-azimuth walkaway VSP data, and such relationships are considered in terms of distance between tensors, as proposed by Gazis et al. (1963). The concept of such a distance is discussed by several researchers, including Norris (2006), Bóna (2009), and Kochetov and Slawinski $(2009 \mathrm{a}, \mathrm{b})$. The present work, which is formulated in the context of a computationally efficient global optimization, allows us to obtain thousands of solutions within a few hours on a multi-core CPU computer. Hence, we can infer properties of materials, together with reliability of such inferences, by examining distributions, illustrated by histograms, of the elasticity parameters of effective tensors and distributions of orientations of these tensors. A computationally efficient scheme is crucial for generating such distributions. 
As discussed by Kochetov and Slawinski (2009a), the distance between a generally anisotropic tensor and its counterpart belonging to a given symmetry class is obtained by finding the orientation that minimizes the distance. Performing a search under all orientations leads to a highly nonlinear optimization problem, which commonly exhibits many local minima. In the past, local-optimization methods have been used, which must be restricted to the vicinity of the global minimum to avoid convergence to a local one. A restriction based on visual examination of distance plots was proposed by Kochetov and Slawinski (2009a, b). Such an examination, however, is practically impossible if we wish to perturb the generally anisotropic tensor thousands of times to consider the effect of errors on the distribution of values that describe properties of effective tensors. Herein, we address this problem by applying a global optimization. Using this method, we can find effective tensors by a Monte Carlo (MC) method (see, e.g., Tarantola 2005), and determine distributions of their orientations and of their elasticity parameters. These distributions arise from errors in which the original tensor is given. That is, our inversion consists of distributions of values that describe properties and orientations of effective tensors. These distributions, which are akin to error bars, allow us to gain an insight into the reliability of a given effective tensor in representing the generally anisotropic one.

This paper has a following layout. First, we review the concept of the effective elasticity tensor and describe the global optimization used in its search. Then, using this optimization, we examine the generally anisotropic tensor obtained by Dewangan and Grechka (2003) and discussed also in Chapter 9 of Tsvankin and Grechka (2011). Next we analyze the sensitivity of the solution to perturbation of elasticity tensor $\mathrm{C}$ using $\mathrm{MC}$ technique. This way we can evaluate the reliability of the solutions.

\section{EFFECTIVE ELASTICITY TENSOR}

For a fixed coordinate system, we can relate a general elasticity tensor, $c$, to its counterpart, $c^{\text {sym }}$, which belongs to a particular symmetry class. Tensor $c^{\text {sym }}$ is the orthogonal projection of $c$, in the sense of the Frobenius inner product (which is the sum of products of the corresponding components, $a_{\mathrm{ijkl}}$ $\left.b_{\mathrm{ijk}}\right)$, onto the linear space containing all tensors of that symmetry class, as described by Gazis et al. (1963).

The distance-squared between $c$ and $c^{\text {sym }}$ is:

$$
d_{\mathrm{sym}}^{2}=\left\|c-c^{\mathrm{sym}}\right\|^{2}=\|c\|^{2}-\left\|c^{\mathrm{sym}}\right\|^{2} .
$$

The second equality is a consequence of the orthogonality of $c$ and $c^{\mathrm{sym}}$. Components of tensor $c^{\text {sym }}$, and, hence, the value of distance obtained from 
Eq. 1, depend on the orientation of the coordinate system. To determine the effective tensor without assuming an a priori orientation, we must minimize $d_{\text {sym }}$ in Eq. 1 with respect to orientations; in other words, we have to perform the minimization of $d_{\text {sym }}$ under all rotations. To find the solution of this high dimensional minimization problem, taking into account an existence of multiple local minima, we formulate a metaheuristic global approach. In this approach, no prior knowledge about a solution is required. The only requirement is that - given two arbitrary points within that space - a candidate for a solution can be chosen based on the difference of value of target function. The manner in which candidates are selected depends on the choice of algorithm. As a search strategy, we choose particle swarm optimization (PSO) because of its simplicity and speed of computation. This search strategy was formulated by Kennedy and Eberhart (1995) and used by Danek et al. (2013) to find the closest tensor of a given symmetry class. Furthermore - unlike other metaheuristics, say, genetic algorithms or simulated annealing - PSO does not require algorithm-parameter tuning (see Donelli et al. 2006). PSO is the stochastic technique that simulates social behavior of animals searching for food, as exemplified by a swarm of fish, insects, etc. In the present case, each particle represents a set of quaternion parameters in a four-dimensional solution space. We choose this representation because quaternions are particularly convenient for describing three-dimensional rotations (see, e.g., Stillwell 2008). In particular, they are computationally more convenient than the Euler angles. During the optimization process, each particle is "aware" of three positions: its current position, $x_{i}$, its best individual position, $p_{i}$, and the best position of the entire swarm, $p_{g}$. Best positions are points in the solution space for which a target function exhibits the lowest value obtained in all previous iterations.

The amplitude of a jump from the previous to the current position is defined by parameter $v_{i}$, called velocity; its value depends on the difference between the best position of an individual particle and the best position found so far by all particles. The canonical PSO formula is (Clerc and Kennedy 2002)

$$
\begin{aligned}
& v_{i}=\chi\left(v_{i}+U\left(0, \Phi_{1}\right) \otimes\left(p_{i}-x_{i}\right)+U\left(0, \Phi_{2}\right) \otimes\left(p_{g}-x_{i}\right)\right), \\
& x_{i}=x_{i}+v_{i}, \\
& \chi=\frac{2}{\Phi-2+\sqrt{\Phi^{2}-4 \Phi}}, \\
& \Phi=\Phi_{1}+\Phi_{2}>4 .
\end{aligned}
$$

where $U$ represents uniform distribution and $\otimes$ is a component wise multiplication. Commonly, $\Phi$, which is the sum of weights of a personal and 
swarm information, $\Phi_{1}$ and $\Phi_{2}$, respectively, is usually set to 4.1 , which means that a constant velocity multiplier, $\chi$, is approximately 0.73 and $U$ is a random number between 0 and approximately 1.5 , if both weights are equal. This scheme guarantees convergences without particle velocity limitations. The flowchart of the algorithm is presented in Fig. 1. Since the elasticity tensor possesses index symmetries, $c_{i j k l}=c_{j i k l}=c_{k l i j}$ (see, e.g., Slawinski 2010), we can write its components as entries of a symmetric $6 \times 6$ matrix. Hence, Hooke's law,

$$
\sigma_{i j}=\sum_{k l=1}^{3} c_{i j k l} \varepsilon_{k l},
$$

can be written in a manner that allows us for a convenient display of elasticity parameters, namely,

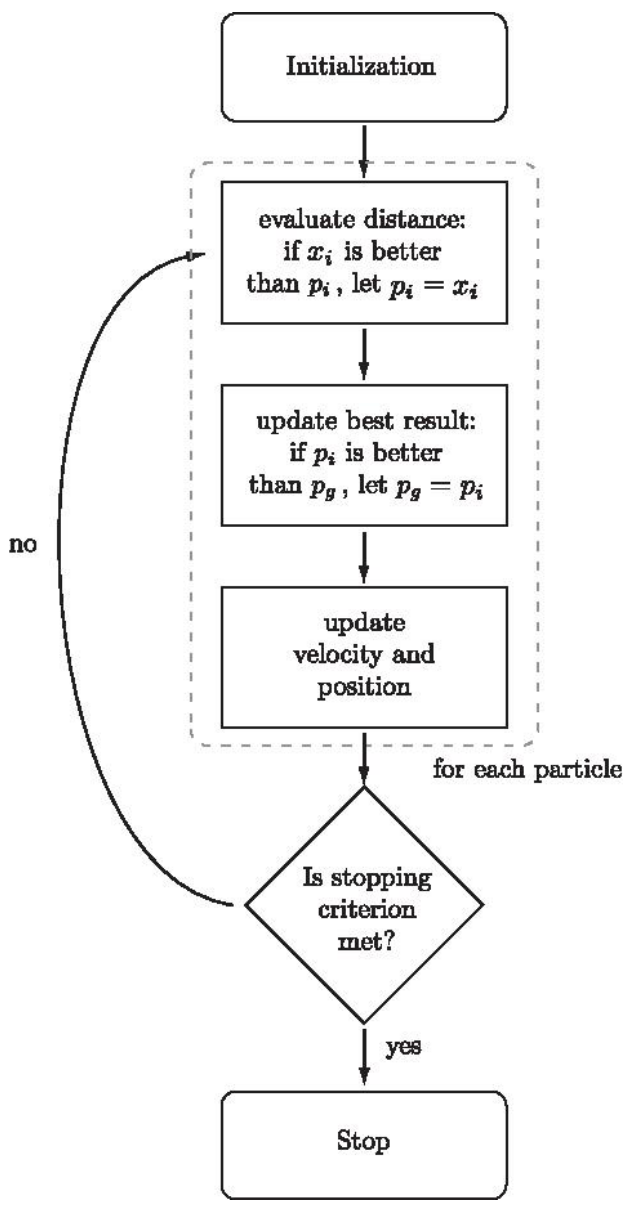

Fig. 1. Flowchart describing the particle-swarm-optimization algorithm: $x_{i}$ is the current particle position, $p_{i}$ is its best position, and $p_{g}$ is the best position for the entire swarm; see text for details about the algorithm. 


$$
\left[\begin{array}{c}
\sigma_{11} \\
\sigma_{22} \\
\sigma_{33} \\
\sqrt{2} \sigma_{23} \\
\sqrt{2} \sigma_{13} \\
\sqrt{2} \sigma_{12}
\end{array}\right]=\left[\begin{array}{cccccc}
c_{1111} & c_{1122} & c_{1133} & \sqrt{2} c_{1123} & \sqrt{2} c_{1113} & \sqrt{2} c_{1112} \\
c_{1122} & c_{2222} & c_{2233} & \sqrt{2} c_{2223} \sqrt{2} c_{2213} \sqrt{2} c_{2212} \\
c_{1133} & c_{2233} & c_{3333} & \sqrt{2} c_{3323} & \sqrt{2} c_{3313} & \sqrt{2} c_{3312} \\
\sqrt{2} c_{1123} & \sqrt{2} c_{2223} & \sqrt{2} c_{3323} & 2 c_{2323} & 2 c_{2313} & 2 c_{2312} \\
\sqrt{2} c_{1113} & \sqrt{2} c_{2213} & \sqrt{2} c_{3313} & 2 c_{2313} & 2 c_{1313} & 2 c_{1311} \\
\sqrt{2} c_{1112} & \sqrt{2} c_{2212} & \sqrt{2} c_{3312} & 2 c_{2312} & 2 c_{1312} & 2 c_{1212}
\end{array}\right]\left[\begin{array}{c}
\varepsilon_{11} \\
\varepsilon_{22} \\
\varepsilon_{33} \\
\sqrt{2} \varepsilon_{23} \\
\sqrt{2} \varepsilon_{13} \\
\sqrt{2} \varepsilon_{12}
\end{array}\right],
$$

which we refer to as the Kelvin notation, we shall denote the elasticity tensor by $C$. Tensor $C$, which includes factors of $\sqrt{2}$ or 2 in its entries, allows us to keep the same norm for both the strain and stress tensors, and as a consequence allows us to conveniently examine rotations associated with symmetry classes (see, e.g., Chapman 2004). Also, unlike the so-called Voigt notation, Eq. 4 is a vector equation. Using the Kelvin notation, we can write the squared distance between $c$ and $c^{\mathrm{sym}}$ as

$$
d_{\text {sym }}^{2}=\|C\|^{2}-\left\|C^{\text {sym }}\right\|^{2},
$$

which is equivalent to Eq. 1.

\section{NUMERICAL STUDY}

The crux for obtaining effective tensors by realizations of a generally anisotropic tensor perturbed by errors relies on the aforementioned global optimization. We apply this method to the tensor obtained from VSP measurements by Dewangan and Grechka (2003):

$$
C=\left[\begin{array}{cccccc}
7.8195 & 3.4495 & 2.5667 & \sqrt{2}(0.1374) & \sqrt{2}(0.0558) & \sqrt{2}(0.1239) \\
3.4495 & 8.1284 & 2.3589 & \sqrt{2}(0.0812) & \sqrt{2}(0.0735) & \sqrt{2}(0.1692) \\
2.5667 & 2.3589 & 7.0908 & \sqrt{2}(-0.0092) & \sqrt{2}(-0.0286) \sqrt{2}(0.1655) \\
\sqrt{2}(0.1374) \sqrt{2}(0.0812) & \sqrt{2}(-0.0092) & 2(1.6636) & 2(-0.0787) & 2(0.1053) \\
\sqrt{2}(0.0558) \sqrt{2}(0.0735) & \sqrt{2}(-0.0286) & 2(-0.0787) & 2(2.0660) & 2(-0.1517) \\
\sqrt{2}(0.1239) & \sqrt{2}(0.1692) & \sqrt{2}(0.1655) & 2(0.1053) & 2(-0.1517) & 2(2.4270)
\end{array}\right]
$$

The components of this generally anisotropic tensor are the densityscaled elasticity parameters; their units are $\mathrm{km}^{2} / \mathrm{s}^{2}$. Entries of matrix 6 were obtained with the following standard deviations: 


$$
S= \pm\left[\begin{array}{cccccc}
0.1656 & 0.1122 & 0.1216 & \sqrt{2}(0.1176) \sqrt{2}(0.0774) \sqrt{2}(0.0741) \\
0.1122 & 0.1862 & 0.1551 & \sqrt{2}(0.0797) \sqrt{2}(0.1137) \sqrt{2}(0.0832) \\
0.1216 & 0.1551 & 0.1439 & \sqrt{2}(0.0856) \sqrt{2}(0.0662) \sqrt{2}(0.1010) \\
\sqrt{2}(0.1176) \sqrt{2}(0.0797) \sqrt{2}(0.0856) & 2(0.0714) & 2(0.0496) & 2(0.0542) \\
\sqrt{2}(0.0774) & \sqrt{2}(0.1137) \sqrt{2}(0.0662) & 2(0.0496) & 2(0.0626) & 2(0.0621) \\
\sqrt{2}(0.0741) & \sqrt{2}(0.0832) \sqrt{2}(0.1010) & 2(0.0542) & 2(0.0621) & 2(0.0802)
\end{array}\right]
$$

Unlike matrixes 6 and 7 does not consist of components of a tensor; it does not satisfy the conditions of tensorial transformations. Thus, $S$ is fixed in the coordinate system in which components 6 are expressed; it can be used as a measure of errors in that system only.

In writing Eqs. 6 and 7, we do not imply that the number of decimal points corresponds to the number of significant digits. We use more decimals to examine numerical stability and to compare accurately our results with those of Kochetov and Slawinski (2009a, b).

To examine the influence of errors, we generate thousands of realizations of tensor 6 with random perturbations whose standard deviations are given in matrix 7. For each realization, using the PSO method, we obtain the effective orthotropic tensor, whose natural orientation is illustrated in Fig. 2, and

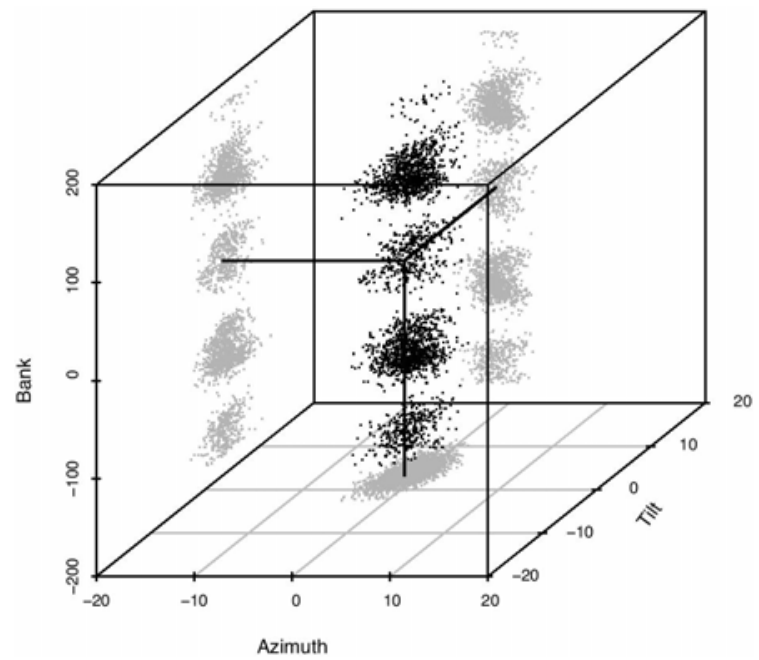

Fig. 2. Four clusters of the effective orthotropic tensors: each black dot is the orientation of the effective tensor corresponding to a realization of tensor 6 subject to errors 7; gray points are projections of black points. The vertex of black lines is the orientation of tensor 8, which results from tensor 6, without errors, and is the closest to the original coordinate system. The axes denote the three Euler angles. Each cluster corresponds to an equally valid natural coordinate system; their slightly different appearances are a result of random perturbation. 
whose axes are the three Euler angles: the azimuth, defined by rotation about the $x_{3}$-axis, the tilt, by rotation about the $x_{1}$-axis, and the bank, by rotation about the new $x_{3}$-axis. Values of these angles are obtained from the quaternions used in the PSO. This figure contains four clusters, which correspond to orientations of natural coordinate systems of orthotropic tensors. Orientations of these systems differ in bank by $\pi / 2$ or its multiple. None of these systems has a privileged status; our choice is a matter of convenience.

\section{EFFECTIVE TENSOR}

\section{Choosing effective tensor}

Since a symmetry class has more than one natural coordinate system - each associated with an aforementioned cluster - for our examinations, we choose systems that are closest to the one in which components (Eq. 6) are expressed. Following the global optimization, components of the effective orthotropic tensor - derived from tensor 6 without errors 7 - in the closest natural coordinate system are

$$
C^{\text {eff }}=\left[\begin{array}{cccccc}
7.7740 & 3.3634 & 2.4276 & 0 & 0 & 0 \\
3.3634 & 8.3762 & 2.4879 & 0 & 0 & 0 \\
2.4276 & 2.4879 & 7.0810 & 0 & 0 & 0 \\
0 & 0 & 0 & 2(1.6497) & 0 & 0 \\
0 & 0 & 0 & 0 & 2(2.0784) & 0 \\
0 & 0 & 0 & 0 & 0 & 2(2.3323)
\end{array}\right]
$$

and the azimuth, tilt, and bank are $-2.4^{\circ}, 2.6^{\circ}$, and $19.3^{\circ}$, respectively. Note the similarity (expected) between tensors 8 and 6 . Also to ensure consistency, note that expression 25 in Kochetov and Slawinski (2009a) and expression 25 in Danek et al. (2013) describe the same effective tensor but stated in a natural coordinate system that, relative to expression 8 , is rotated by $\pi / 2$ about the new $x_{3}$-axis.

According to the work of Dewangan and Grechka (2003) and Kochetov and Slawinski (2009a), tensor 6 can be represented by its counterpart exhibiting orthotropic symmetry. Let us use our method to examine whether or not a lesser or greater symmetry is a good representation of tensor 6 subject to errors 7.

Using the aforementioned global optimization, we obtain the distribution of shortest distances of tensor 6 to effective tensors of monoclinic, orthotropic, transversely isotropic, and isotropic symmetries. We compare these distributions to the distribution of the Frobenius norm of errors 7, which we obtain by generating random realizations of a zero tensor with these errors. This operation results in a distribution that is a square-root of the sum of thirty-six squares of independent random variables, $M_{i j}$, having a normal dis- 
tribution with the zero mean and standard deviations given in matrix 7 . Even though this distribution could be obtained analytically (see, e.g., Mathai and Provost 1992), we use the same numerical method that we use to obtain the distributions of distances of tensor 6 to effective tensors.

To clarify that the expected value of the norm of

$$
\left[M_{i j}\right]=\left[\begin{array}{ccc}
N\left(0, S_{11}^{2}\right) & \cdots & N\left(0, S_{16}^{2}\right) \\
\vdots & \ddots & \vdots \\
N\left(0, S_{61}^{2}\right) & \cdots & N\left(0, S_{66}^{2}\right)
\end{array}\right],
$$

where $N\left(0, S_{i j}^{2}\right)$ are random variables and $S_{i j}$ are entries of matrix 7 , is not equal to zero, let us consider the variance

$$
\operatorname{Var}(X):=E\left[X^{2}\right]-(E[X])^{2},
$$

where $X$ is a random variable and $E$ denotes the expected value. In our case, $E[X]=0$, so the expected value of the square of random variable is equal to its variance. Hence, the expected value of the norm of matrix 9 is

$$
E(|M|)=\sqrt{\sum_{i, j=1}^{6} S_{i j}^{2}},
$$

which is the norm of matrix 7, namely, 0.7844 . This value is in agreement with the value obtained numerically: 0.7747 , which corresponds to the location of the apex of the black line in Fig. 3.

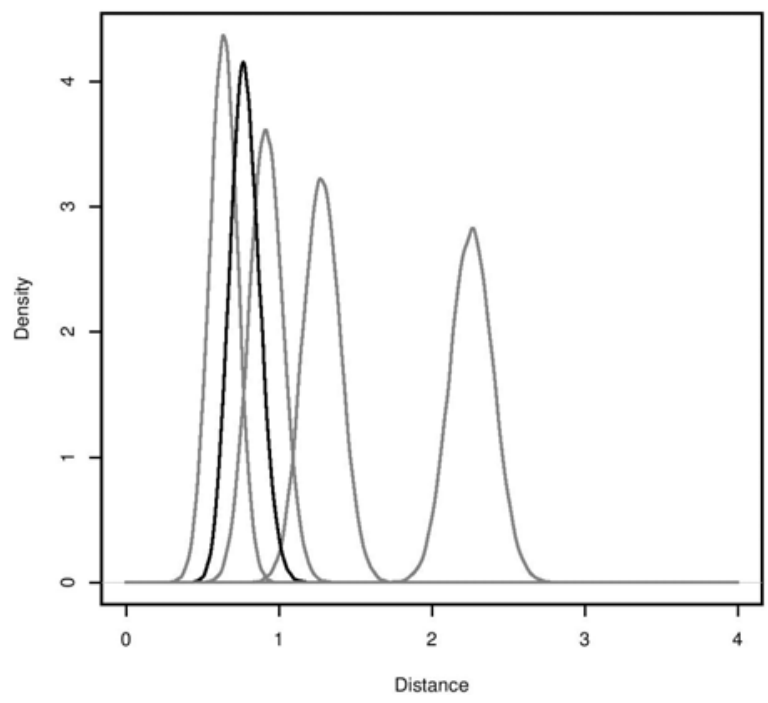

Fig. 3. Density distributions: the black line represents density of the norm of matrix 9 , whose mean value - by expression 11 - is equal to the norm of matrix 7. Proceeding from left to right, gray lines represent densities of distance distributions for monoclinic, orthotropic, transversely isotropic, and isotropic symmetries, respectively. 
In Figure 3, the distribution of errors 7 overlaps not only with the distribution of distances to the orthotropic symmetry but also to the monoclinic and transversely isotropic symmetries. As suggested by Kochetov and Slawinski (2009b), we view the overlap between the distribution of the distance from tensor 6 with its symmetric counterpart and with the distribution of the Frobenius norm of errors 7 as an indication that a symmetric tensor might represent tensor 6 . Hence, the following question arises: could we choose a tensor of monoclinic or transversely isotropic symmetry to represent tensor 6 ?

To address this question, we note that the natural-coordinate expressions of the orthotropic, and higher, symmetries require $c_{1112}=c_{2212}=c_{3313}=0$ (see, e.g., Slawinski 2010). For examination of this issue, we generate thousands of realizations of tensor 6 subject to errors 7 , and express them in the orientations of their closest monoclinic counterparts. Since $(0,0,0)$ is in the center of the obtained cluster shown in Fig. 4, we conclude that tensor 6 with errors 7 appears to be more symmetric than monoclinic.

Also, the natural-coordinate expressions of transverse isotropy require $c_{1111}=c_{2222}, c_{1133}=c_{2233}$, and $c_{2323}=c_{1313}$. To examine this issue, we generate thousands of realizations and express them in the orientations of their closest transversely isotropic counterparts. Examining the left panels in

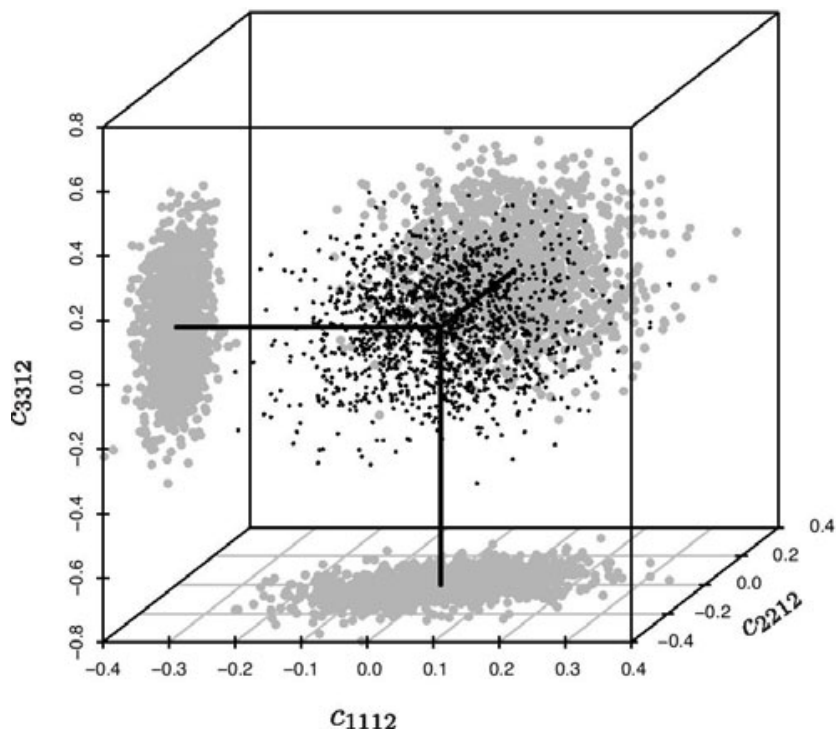

Fig. 4. Selected entries of realizations of tensor 6 subject to errors 7 in coordinate systems whose orientations correspond to the closest monoclinic tensors. Note that with no constraints applied - the values of $c_{1112}, c_{2212}$, and $c_{3313}$ are scattered around zeros, which are highlighted by solid lines. From this pattern we infer that tensor 6 can be represented by an effective tensor whose symmetry is higher than monoclinic. 

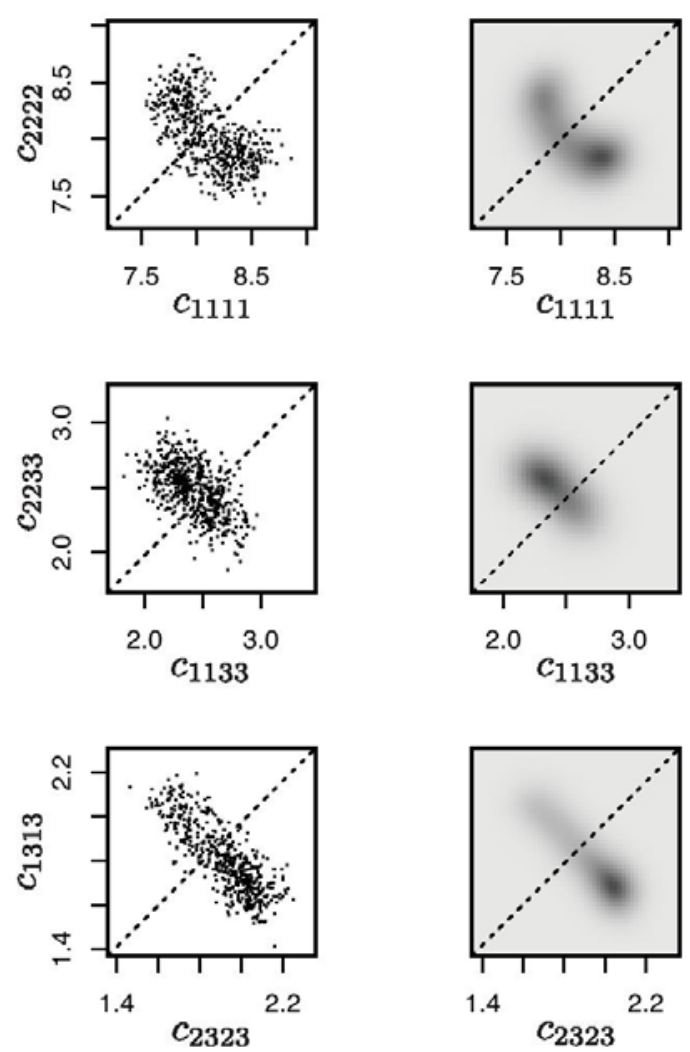

Fig. 5. Selected entries of realizations of tensor 6 subject to errors 7 expressed in coordinate systems whose orientations correspond to the closest transversely isotropic tensors, and their marginal distributions. For picture clarity, only 1000 points are shown on the left panel.

Fig. 5, we observe that obtained clusters are crossed by dashed lines showing these equalities; however, from examination of the right panel, we see that two-dimensional marginal distributions show that areas of the highest density are away from these equalities. We conclude that tensor 6 with errors 7 exhibits a lesser symmetry than transverse isotropy.

Thus, we choose the orthotropic symmetry to represent tensor 6 .

\section{Properties of chosen effective tensor}

Having accepted that tensor 6 subject to errors 7 can be represented by an effective orthotropic tensor, let us examine its properties in the context of errors. These properties are the elasticity parameters, whose distributions are illustrated in Fig. 6, and orientation, whose distribution is illustrated in Fig. 7. 

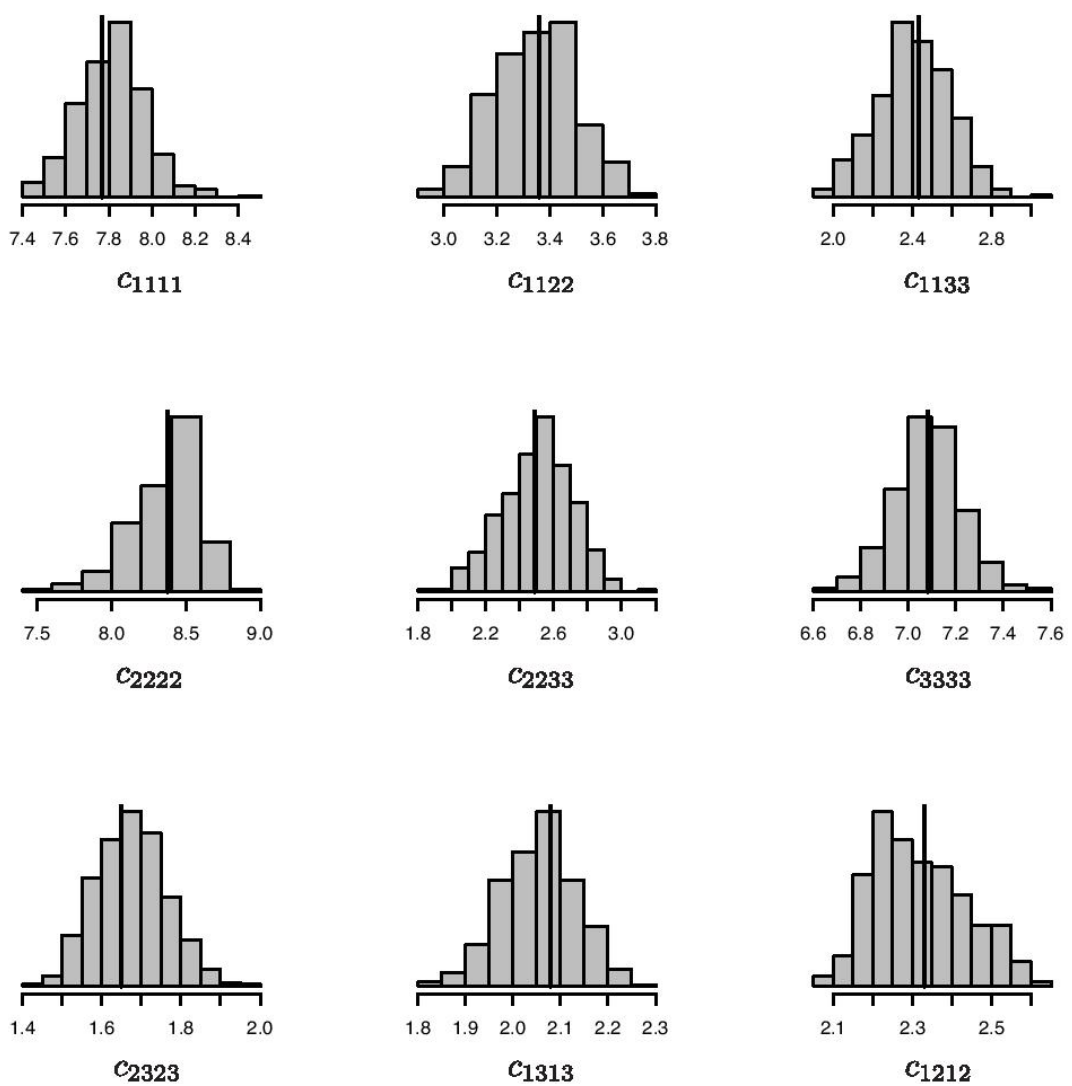

Fig. 6. Histograms of density-scaled elasticity parameters of the effective orthotropic tensors obtained from realizations of tensor 6 , subject to errors 7 , in natural coordinates whose orientations are illustrated in Fig. 7. Solid lines correspond to the values for the error-free case.

Furthermore, from the properties of this tensor we can infer properties of materials examined by VSP measurements from which tensor 6 is obtained.

The behavior of the histograms in Fig. 6, including their unimodality and relatively confined widths, suggests that the orthotropic symmetry contains much information about tensor 6 subject to errors 7, which is consistent with the symmetry choice discussed above. Examining the azimuth and tilt displayed in Fig. 7, we see that one of the symmetry planes of the effective orthotropic tensor is close to horizontal. The value of the bank indicates that the axes of the system in which tensor 6 is expressed are oblique to natural coordinates of the effective tensor. This information can be used to infer orientations of layers and fractures in a manner akin to those examined by Grechka and Kachanov (2006). 

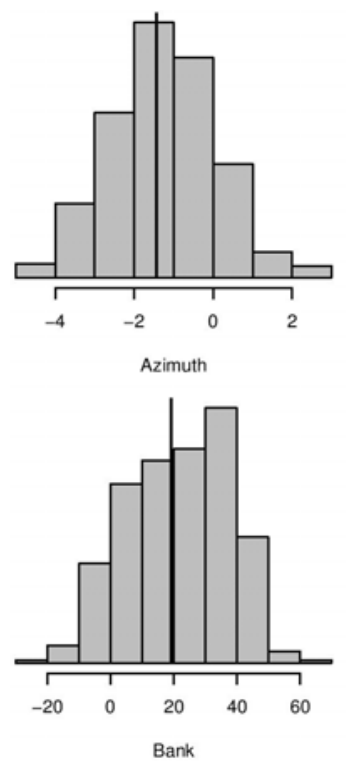
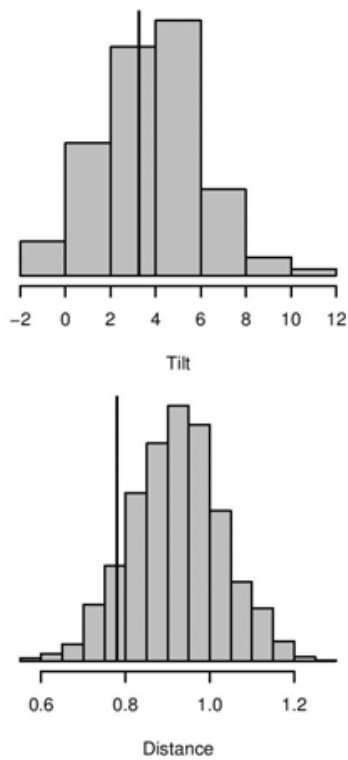

Fig. 7. Histograms of the azimuth, tilt, bank (in degrees), and distance (in $\mathrm{km}^{2} / \mathrm{s}^{2}$ ), from tensor 6 to effective orthotropic tensors obtained from realizations of tensor 6 subject to errors 7. The first three histograms are projections of the cluster in Fig. $2-$ whose bank is close to zero - to the three axes therein. Solid lines correspond to the values for the error-free case.

To gain an insight into the strength of anisotropy under consideration, following the formulation proposed by Tsvankin (1997) let us express the pertinent components of tensor 8 in terms of the seven parameters that are zero for the case of isotropy. Our results shown in Fig. 8 are consistent with those presented by Dewangan and Grechka (2003) and further elaborated by Tsvankin and Grechka (2011), Section 7.13.

As shown in Fig. 8, distributions of several among the aforementioned parameters contain zero. Nevertheless, absolute average values of $\delta^{(1)}$ and $\gamma^{(2)}$ are close to 0.2 , which suggests that anisotropy is not weak. Moreover, the shear-wave-splitting coefficient, $\left(c_{1313}-c_{2323}\right) /\left(2 c_{2323}\right)$, which is important in fracture detection, is about 0.12; again, it is similar to 0.1 obtained by Tsvankin and Grechka (2011). This value is relatively large since, typically, the observed splitting coefficients are less than 0.05 (Tsvankin 2013, pers. comm.).

Note that values of $i$ in $\delta^{(i)}, \varepsilon^{(i)}$, and $\gamma^{(i)}$, in this figure, are interchanged with respect to values in Kochetov and Slawinski (2009b) because the coordinate systems differ by $\pi / 2$ about the new $x_{3}$-axis. Similarly, the interchange in Dewangan and Grechka (2003) and Tsvankin and Grechka (2011) is a consequence of coordinate systems belonging to different clusters shown in 


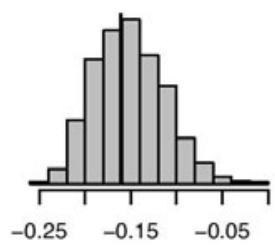

$\delta^{(1)}$

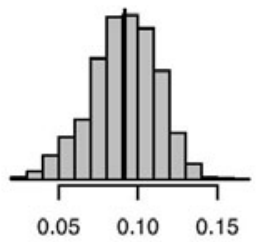

$\epsilon^{(1)}$

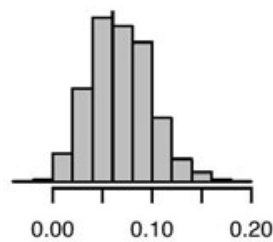

$\gamma^{(1)}$

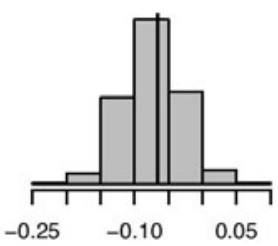

$\delta^{(2)}$

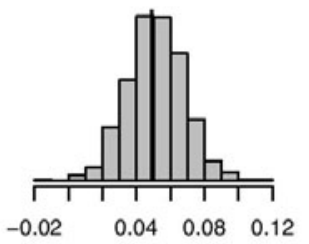

$\epsilon^{(2)}$

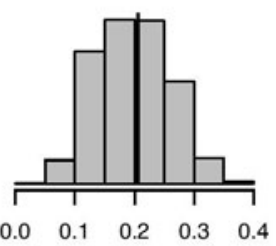

$\gamma^{(2)}$

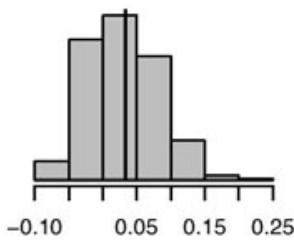

$\delta^{(3)}$

Fig. 8. Histograms of the seven elasticity parameters of orthotropic symmetry, whose values are zero in the case of isotropy. Solid lines correspond to the values for the error-free case.

Fig. 2. None of the clusters is privileged, as long as all results are expressed with respect to the same system.

\section{CONCLUSIONS}

The presented method allows us to infer from seismic measurements information about materials represented by a generally anisotropic tensor. This method extends the approach introduced by Kochetov and Slawinski (2009a) in two important ways.

First, as discussed by Danek et al. (2013), it invokes a global optimization method, which allows us to directly consider tensors of all symmetry classes, regardless of their orientation being described by two or three Euler angles. We note that, as discussed by Kochetov and Slawinski (2009a,b), constraining the local search to obtain absolute minima requires an examination that is possible only for tensors whose orientations are described by only two Euler angles; such tensors are either monoclinic or transversely isotropic. 
Second, this direct approach allows us to perturb the values of the original tensor thousands of times to obtain estimates of its effective tensors in the presence of errors. Hence, by using the Monte-Carlo approach, we can estimate ranges of values for elasticity parameters and orientations of effective tensors for cases such as that represented by tensor 6 with errors 7 .

Within the assumption of a normal distribution of errors, one could also examine the best fit in terms of likelihood by including errors in the distance function, as considered by Bóna (2009). We use the same errors to perturb the original tensor and - while remaining within coordinate-invariant definition of distance - obtain distributions of solutions, within whose range we would find the effective tensor obtained by the approach of Bóna (2009).

In this study, we confirm and further quantify conclusions obtained originally by Dewangan and Grechka (2003) and examined also by Kochetov and Slawinski (2009a) about the symmetry, orientation, and component values of tensor 8. In particular, we conclude that tensor 6 with errors 7 is consistent - in the Monte-Carlo sense - with the orthotropic symmetry class. Also the results of this paper are consistent with comments of Grechka and Kachanov (2006), according to whom orthotropy might suffice for many scenarios encountered in exploration seismology.

Acknowledgments. This work was inspired by collaboration with the late Albert Tarantola. Also, the authors acknowledge discussions with, and fruitful suggestions of Misha Kochetov, Ken Larner, Daniel Peter, Michael Rochester and Ilya Tsvankin, editorial help of David Dalton and graphic support of Elena Patarini. TD received funding from the Atlantic Innovation Fund and the Research and Development Corporation of Newfoundland and Labrador through the High Performance Computing for Geophysical Applications Project and from Polish National Science Center through grant number 2011/01/B/ST10/07305. MS's research was partially supported by the Discovery Grant of The Natural Sciences and Engineering Research Council of Canada. This research was performed in the context of The Geomechanics Project supported by Husky Energy.

References

Bóna, A. (2009), Symmetry characterization and measurement errors of elasticity tensors, Geophysics 74, 5, 75-78, DOI: 10.1190/1.3184013.

Chapman, C. (2004), Fundamentals of Seismic Wave Propagation, Cambridge University Press, Cambridge. 
Clerc, M., and J. Kennedy (2002), The particle swarm - explosion, stability, and convergence in a multidimensional complex space, IEEE Trans. Evolut. Comp. 6, 1, 58-73, DOI: 10.1109/4235.985692.

Danek, T., M. Kochetov, and M.A. Slawinski (2013), Uncertainty analysis of effective elasticity tensors using quaternion-based global optimization and Monte-Carlo method, Q. J. Mech. Appl. Math. 66, 2, 253-272, DOI: 10.1093/qjmam/hbt004.

Dewangan, P., and V. Grechka (2003), Inversion of multicomponent, multiazimuth, walkawayVSP data for the stiffness tensor, Geophysics 68, 3, 1022-1031, DOI: $10.1190 / 1.1581073$.

Donelli, M., G. Franceschini, A. Martini, and A. Massa (2006), An integrated multiscaling strategy based on a particle swarm algorithm for inverse scattering problems, IEEE Trans. Geosci. Remote Sens. 44, 2, 298-312, DOI: 10.1109/TGRS. 2005.861412.

Gazis, D.C., I. Tadjbakhsh, and R.A. Toupin (1963), The elastic tensor of given symmetry nearest to an anisotropic elastic tensor, Acta Crystallogr. 16, 9, 917-922, DOI: 10.1107/S0365110X63002449.

Grechka, V., and M. Kachanov (2006), Seismic characterization of multiple fracture sets: Does orthotropy suffice? Geophysics 71, 3, D93-D105, DOI: 10.1190/ 1.2196872 .

Kennedy, J., and R. Eberhart (1995), Particle swarm optimization. In: Proc. IEEE Int. Conf. Neural Networks, 27 November - 1 December 1995, Perth, Australia, 1942-1948.

Kochetov, M., and M.A. Slawinski (2009a), Estimating effective elasticity tensors from Christoffel equations, Geophysics 74, 5, 67-73, DOI: 10.1190/ 1.3155163 .

Kochetov, M., and M.A. Slawinski (2009b), On obtaining effective orthotropic elasticity tensors, Q. J. Mech. Appl. Math. 62, 2, 149-166, DOI: 10.1093/ qjmam/hbp001.

Mathai, A.M., and S.B. Provost (1992), Quadratic Forms in Random Variables: Theory and Applications, Statistics: Textbooks and Monographs, Vol. 126, Dekker, New York.

Norris, A.N. (2006), The isotropic material closest to a given anisotropic material, J. Mech. Mater. Struct. 1, 2, 223-238, DOI: 10.2140/jomms.2006.1.223.

Slawinski, M.A. (2010), Waves and Rays in Elastic Continua, World Scientific Publ., Singapore.

Stillwell, J. (2008), Naive Lie Theory. Undergraduate Texts in Mathematics, Springer, New York, DOI: 10.1007/978-0-387-78214-0.

Tarantola, A. (2005), Inverse Problem Theory and Methods for Model Parameter Estimation, SIAM, Philadelphia.

Tsvankin, I. (1997), Anisotropic parameters and P-wave velocity for orthorhombic media, Geophysics 62, 4, 1292-1309, DOI: 10.1190/1.1444231. 
Tsvankin, I., and V. Grechka (2011), Seismology of Azimuthally Anisotropic Media and Seismic Fracture Characterization, Geophysical References Series, Society of Exploration Geophysicists, DOI: 10.1190/1.9781560802839.

Received 30 July 2013

Received in revised form 7 October 2013

Accepted 14 October 2013 